\title{
Relações entre saúde mental e falhas cognitivas no dia a dia: papel dos sintomas internalizantes e externalizantes
}

\author{
Relationship between mental health and everyday cognitive \\ failures: role of internalizing and externalizing symptoms
}

Aline Alves Ferreira, , Wanessa Gabrielli Augusto Oliveira,2, Jonas Jardim de Paula $a^{1,2,3}$

\section{RESUMO}

Objetivo: Falhas cognitivas são erros cometidos no dia a dia como perda de compromissos, falta de palavras ao conversar, dificuldades de memorizar o que é lido, erros de orientação, entre outros. Sintomas psiquiátricos internalizantes e externalizantes podem contribuir para a ocorrência de falhas cognitivas. O presente estudo objetiva avaliar como sintomas internalizantes e externalizantes contribuem para a ocorrência de falhas cognitivas no dia a dia. Métodos: Foram avaliados 366 voluntários utilizando o Questionário de Falhas Cognitivas (CFQ) e as escalas Self-Reporting Questionnaire-20 (SRQ-20), como medida de sintomas internalizantes, e Adult Self-Report Scale (ASRS-18), como medida de sintomas externalizantes. Os participantes foram classificados com base nos pontos de corte da SRQ-20 e ASRS-18 para a criação de quatro grupos: hígido, internalizante, externalizante e misto. Comparamos os grupos por meio de ANOVA e testamos a associação das variáveis estudadas por meio de correlações. Resultados: Todas as escalas apresentaram correlações fortes entre si ( $r>0,500$, $p<0,001)$. A comparação dos grupos sugere que participantes com escores clínicos de sintomas internalizantes e externalizantes apresentam mais falhas quando comparados ao grupo hígido ( $d=0,86$ e $d=1,00$ ), mas não apresentam diferenças entre si $(d=0,21)$. Participantes do grupo misto apresentaram mais falhas que o grupo hígido $(d=2,01)$, internalizante $(d=1,31)$ e externalizante $(d=1,05)$. Conclusão: Tanto sintomas internalizantes quanto externalizantes contribuem para a ocorrência de falhas cognitivas no dia a dia. Observamos ainda um efeito aditivo de ambos os sintomas.

\section{ABSTRACT}

Objective: Cognitive failures are errors made in everyday life such as forgetting appointments, lack of words when talking, difficulties to memorize what is read, errors of orientation, among others. Internalizing and externalizing psychiatric symptoms may contribute to the occurrence of cognitive failures. The present study aims to evaluate how internalizing and externalizing symptoms contribute to the occurrence of everyday cognitive failures. Methods: We evaluated 366 volunteers using the Cognitive Failure Questionnaire (CFQ), the Self-Reporting Questionnaire-20 (SRQ-20) as a measure of internalizing symptoms and the

1 Faculdade de Ciências Médicas de Minas Gerais, Departamento de Psicologia, Belo Horizonte, MG, Brasil.

2 Laboratório de Ensino e Pesquisa em Neuropsicologia (Labep Neuro), Belo Horizonte, MG, Brasil.

3 Universidade Federal de Minas Gerais (UFMG), Laboratório de Neurociência e Medicina Molecular, Belo Horizonte, MG, Brasil. 


\section{Keywords}

Depression, attention deficit hyperactivity disorder, memory, attention, executive function.
Adult Self-Report Scale (ASRS-18) as a measure of externalizing symptoms. We classified the participants based on the SRQ-20 and ASRS-18 cut-off scores to create four groups: controls, internalizing, externalizing, and mixed. We compared the groups by means of ANOVA and tested its associations with correlations. Results: All scales showed strong correlations between each other $(r>0.500, p<0.001)$. The comparison of the groups suggests that participants with clinical scores of internalizing and externalizing symptoms have more failures when compared to the control group ( $d=0.86$ and $d=1.00$ ), but they do not present differences between themselves $(d=0.21)$. Participants of the mixed group presented more failures than the control group $(d=2.01)$, and the internalizing $(d=1.31)$ and externalizing $(d=1.05)$ groups. Conclusion: Both internalizing and externalizing symptoms contribute to the occurrence of day-to-day cognitive failures. We also observed an additive effect of both symptoms.

\section{INTRODUÇÃO}

A cognição humana pode ser descrita como um agrupamento de habilidades responsáveis pelo aprendizado, compreensão, integração e aplicação de informações variadas que ocorrem no cotidiano. Essas habilidades podem ser agrupadas de form-a geral em cinco grandes construtos: funções executivas, linguagem, atenção, processamento espacial e memória, segundo evidências da neuropsicologia e da psicologia das diferenças individuais?'.

Erros cognitivos no dia a dia são comumente reconhecidos como falhas cognitivas ${ }^{2}$. Falhas cognitivas são pequenos erros de memória, percepção, planejamento e execução de determinada tarefa ou atividade, sendo frequentes no cotidiano da maioria das pessoas. Essas falhas raramente refletem o funcionamento cognitivo em si, em termos de "traço", mas em geral representam problemas na aplicação dessas funções em situações do cotidiano, pouco estruturadas, sendo melhor compreendidas em termos de um "estado" cognitivo ${ }^{3}$. Essas falhas cognitivas podem ser consideradas comuns entre adultos hígidos, sendo relacionadas a fatores como maior exposição ao estresse, fatores de personalidade, sono ou com a cognição ${ }^{3}$. A ocorrência de falhas cognitivas no cotidiano pode ser um indicativo de transtornos mentais, ocorrendo com frequência em transtornos internalizantes e externalizantes ${ }^{2,4}$. As consequências negativas das falhas cognitivas no dia a dia não se restringem à frustração ou ao sofrimento psicológico, mas comprometem o funcionamento social do indivíduo, por exemplo, em seu desempenho escolar 5 .

De forma geral, sintomas típicos dos transtornos mentais mais comuns podem ser agrupados em dois grandes grupos: internalizantes - disforia, anedonia, retraimento social, ansiedade e sintomas somáticos - e externalizantes - impulsividade, uso de substâncias, hiperatividade, desatenção e agressividade ${ }^{6}$. Ambos os sintomas podem influenciar o funcionamento cognitivo e seus reflexos no dia a dia ${ }^{7,8}$. Os sintomas de disforia e anedonia são considerados critérios diagnósticos centrais ao transtorno depressivo maior, enquanto outros sintomas internalizantes são considerados critérios secundários. Sintomas depressivos relacionados ao polo de anedonia, como a apatia e a lentificação psicomotora, são associados a pior funcionalidade no dia a dia e a maior demanda por suporte externo na realização de atividades rotineiras ${ }^{9}$. Por outro lado, os sintomas de desatenção, hiperatividade e impulsividade são centrais ao transtorno do déficit de atenção e hiperatividade (TDAH). Esse transtorno do neurodesenvolvimento cursa comumente com comprometimento das funções executivas e da atenção, comprometimento esse que se reflete negativamente no dia a dia ${ }^{10}$. Ainda que conceitualmente sintomas externalizantes e internalizantes sejam construtos independentes, uma sobreposição importante ocorre entre ambos, como observado, por exemplo, na maior ocorrência de sintomas depressivos em pessoas com TDAH ${ }^{11}$ ou na maior expressão de impulsividade em pacientes com transtorno depressivo maior ${ }^{12}$. Nesse sentido, tanto os sintomas externalizantes quanto os internalizantes podem influenciar as falhas cognitivas no dia a dia. Nesse sentido, uma associação de ambos os tipos de sintoma com as falhas cognitivas poderia sugerir que essas falhas apresentam múltiplas etiologias, em que diferentes quadros clínicos acarretariam sintomas semelhantes. Esse tópico é pouco investigado na literatura, embora uma revisão sobre falhas cognitivas em adultos sugira uma etiologia multidimensional, embora os estudos em geral não investiguem essa hipótese ${ }^{3}$. Não se sabe, contudo, como esses dois tipos de sintomas interagem para a ocorrência das falhas cognitivas no dia a dia.

Diante da possível associação de sintomas internalizantes e externalizantes com as falhas cognitivas no dia a dia, o objetivo do estudo é avaliar como esses três fatores se relacionam na população adulta. Abordaremos essa pergunta tanto em uma perspectiva categórica/clínica (considerando participantes com sintomas em intensidade atipicamente alta) quanto em uma perspectiva dimensional (considerando a intensidade dos sintomas em si). Nossa hipótese é que tanto sintomas internalizantes quanto externalizantes contribuirão para a ocorrência de falhas cognitivas. 


\section{MÉTODOS}

\section{Participantes}

Esta pesquisa faz parte de um projeto mais abrangente, aprovado pelo comitê de ética da Faculdade de Ciências Médicas de Minas Gerais (CAAE: 57378016.5.0000.5134). Participaram deste estudo 366 adultos brasileiros. A coleta de dados foi realizada por meio da internet, em uma plataforma virtual construída pelos pesquisadores utilizando o aplicativo Google Forms. Estudos anteriores sugerem boa concordância entre a coleta de dados via internet e o uso de questionários e escalas em formato tradicional ${ }^{13,14}$. Esse tamanho amostral permite a detecção de efeitos moderados, com poder amostral variando de $98 \%$ a $99 \%$ em nosso estudo, dependendo das técnicas utilizadas (essa estimativa foi realizada por meio software $\mathrm{G}^{*}$ Power ${ }^{15}$ ). A descrição dos participantes é apresentada na Tabela 1.

Tabela 1. Descrição dos participantes

\begin{tabular}{|c|c|c|c|}
\hline & & $\mathrm{n}$ & $\%$ \\
\hline \multirow[t]{3}{*}{ Idade } & $18-24$ anos & 186 & $51 \%$ \\
\hline & 25-34 anos & 117 & $32 \%$ \\
\hline & $35+$ anos & 63 & $17 \%$ \\
\hline \multirow[t]{2}{*}{ Sexo } & Masculino & 129 & $35 \%$ \\
\hline & Feminino & 237 & $65 \%$ \\
\hline \multirow[t]{4}{*}{ Estado civil } & Sem informação & 11 & $3 \%$ \\
\hline & Casado & 78 & $21 \%$ \\
\hline & Solteiro & 268 & $73 \%$ \\
\hline & Divorciado & 9 & $2 \%$ \\
\hline \multirow[t]{3}{*}{ Educação } & Básica & 15 & $4 \%$ \\
\hline & Colegial & 201 & $55 \%$ \\
\hline & Superior & 150 & $41 \%$ \\
\hline \multirow[t]{2}{*}{ Tipo de escola } & Privada & 161 & $44 \%$ \\
\hline & Pública & 205 & $56 \%$ \\
\hline \multirow[t]{4}{*}{ Ocupação } & Não estuda ou trabalha & 19 & $5 \%$ \\
\hline & Estuda & 125 & $34 \%$ \\
\hline & Trabalha & 96 & $26 \%$ \\
\hline & Estuda e Trabalha & 126 & $34 \%$ \\
\hline \multirow[t]{2}{*}{ Histórico de transtorno mental } & Não & 290 & $79 \%$ \\
\hline & Sim & 76 & $21 \%$ \\
\hline \multirow[t]{6}{*}{ Transtornos mentais relatados } & TDAH & 12 & $3 \%$ \\
\hline & Ansiedade & 10 & $3 \%$ \\
\hline & Depressão & 30 & $8 \%$ \\
\hline & Depressão e ansiedade & 7 & $2 \%$ \\
\hline & Transtorno do pânico & 10 & $3 \%$ \\
\hline & Outro & 5 & $1 \%$ \\
\hline \multirow{2}{*}{$\begin{array}{l}\text { Uso de medicamento } \\
\text { psicotrópico }\end{array}$} & Não & 319 & $87 \%$ \\
\hline & Sim & 47 & $13 \%$ \\
\hline
\end{tabular}

TDAH: transtorno do déficit de atenção e hiperatividade.

\section{Avaliação dos participantes Questionário de Falhas Cognitivas (QFC) ${ }^{2}$}

O QFC é um instrumento de autorrelato contendo 25 questões sobre falhas cognitivas cometidas por adultos no dia a dia. O instrumento foi padronizado para uso clínico ${ }^{15}$ e validado para o contexto brasileiro ${ }^{16}$. O questionário possui aplicação breve e relativamente simples e fornece uma variável de natureza ecológica, representando a frequência, intensidade e padrão de falhas cognitivas que o sujeito apresenta no cotidiano. O QFC envolve perguntas como "Você lê alguma coisa, percebe que não estava prestando atenção e precisa lê-la novamente?", "Você esquece onde colocou alguma coisa, como o jornal, o celular ou suas chaves?" e "Você confunde esquerda e direita ao indicar o caminho a alguém?", e o sujeito responde em uma escala de cinco pontos, variando de nunca a quase sempre. A confiabilidade do QFC é superior a 0,916. Os resultados variam de 0 a 100. Escores mais elevados indicam maior ocorrência de falhas cognitivas.

\section{Self-Reporting Questionnaire (SRQ-20).}

O SQR-20 é uma escala para rastreio de transtornos mentais não psicóticos, com foco em sintomas internalizantes. As respostas são do tipo sim/não. Cada resposta afirmativa é pontuada com o valor de 1 para compor o escore final por meio do somatório desses valores. Os escores variam de 0 a 20, sendo os escores mais elevados relacionados à maior intensidade de sintomas. A escala apresenta boa confiabilidade, com consistência interna superior a 0,817. No Brasil, o estudo proposto por Gonçalves et al. ${ }^{18}$ apresenta o ponto de corte 7/8 (não clínico/clínico) como o mais preciso para a detecção de transtornos de natureza internalizante.

\section{Adult Self-Report Scale (ASRS-18)}

Trata-se de uma escala que avalia sintomas de desatenção, hiperatividade e impulsividade, aspectos centrais do TDAH e outros transtornos externalizantes. As respostas são pontuadas com base na frequência com a qual os sintomas se apresentam no dia a dia. Pontuam-se separadamente as dimensões desatenção e hiperatividade/impulsividade, e os escores variam entre 0 e 36 pontos para cada uma delas. Escores mais elevados são indicativos de maior número de sintomas ${ }^{19}$. A confiabilidade da escala é superior a 0,920. No Brasil, o estudo proposto por Leite ${ }^{20}$ sugere que escores iguais ou superiores a 21 em qualquer uma das duas dimensões classificam corretamente $94 \%$ dos adultos com TDAH quando comparados a controles típicos.

\section{Análise estatística}

Após análise da distribuição dos dados, avaliamos a associação de sintomas externalizantes, internalizantes e falhas cognitivas por meio de correlações de Pearson, conduzidas na amostra como um todo, e o teste $Z$ de Steiger, para compa- 
rar a magnitude das correlações. Posteriormente, dividimos os participantes em quatro grupos, com base nos pontos de corte reportados anteriormente para a SRQ-20 e a ASRS-18: hígidos/controles (abaixo do ponto de corte de ambas as escalas), internalizante (acima do ponto de corte da SRQ-20, mas não da ASRS-18), externalizante (acima do ponto de corte na ASRS-18, mas não da SRQ-20) e misto (acima do ponto de corte tanto na ASRS-18 quanto na SRQ-20). Comparamos os quatro grupos em termos de falhas cognitivas por meio de análise de variância simples, com comparações post hoc ajustadas pelo método de Bonferroni. Os procedimentos foram realizados no software Jasp $0.8^{21}$.

\section{RESULTADOS}

As correlações entre as falhas cognitivas, sintomas internalizantes e externalizantes são apresentadas na Tabela 2. Todas as correlações foram significativas ( $p<0,001)$, positivas e fortes $(r>0,500)$. Nota-se que os sintomas de desatenção foram os mais fortemente associados ao CFQ quando comparados sintomas internalizantes $(p<0,001)$ e os de hiperatividade-impulsividade $(p<0,001)$.

Tabela 2. Correlações entre as falhas cognitivas (CFQ), sintomas internalizantes (SRQ-20) e sintomas externalizantes (ASRS-18)

\begin{tabular}{lcccc}
\hline & CFQ & SRQ-20 & ASRS-18 (HI) & ASRS-18 (D) \\
\hline CFQ & - & $0.589^{* *}$ & $0.513^{* *}$ & $0.760^{* *}$ \\
SRQ-20 & - & $0.446^{* *}$ & $0.527^{* *}$ \\
ASRS-18 (HI) & & & - & $0.506^{* *}$ \\
ASRS-18 (D) & & & & - \\
\hline
\end{tabular}

** $\mathrm{p}<0,001$; CFQ: Questionário de Falhas Cognitivas; SRQ-20: Self-Reporting Questionnaire 20; ASRS-18: Adult Self-Reported Scale 18; HI: hiperatividade-impulsividade; D: desatenção.

A Tabela 3 apresenta a pontuação média e a variabilidade dos resultados nas escalas adotados no estudo, estratificadas pelos quatro grupos criados anteriormente. Observa-se que, independentemente do grupo, o relato da ocorrência de falhas cognitivas é comum, visto que a média do grupo hígido é equivalente a aproximadamente um terço do total do questionário.

Tabela 3. Descrição dos participantes nos questionários adotados no estudo

\begin{tabular}{lcccc}
\hline & $\begin{array}{c}\text { Controle } \\
(\mathbf{n}=229)\end{array}$ & $\begin{array}{c}\text { Internalizante } \\
(\mathbf{n}=64)\end{array}$ & $\begin{array}{c}\text { Externalizante } \\
(\mathbf{n}=26)\end{array}$ & $\begin{array}{c}\text { Misto } \\
(\mathbf{n}=47)\end{array}$ \\
\cline { 2 - 5 } & $\mathbf{M}(\mathbf{D P})$ & $\mathbf{M}(\mathbf{D P})$ & \multicolumn{1}{c}{$\mathbf{M}(\mathbf{D P})$} & $\mathbf{M}(\mathbf{D P})$ \\
\hline Idade & $29,10(10,49)$ & $25,98(8,38)$ & $24,88(6,79)$ & $25,53(7,56)$ \\
CF0 & $35,72(12,09)$ & $45,92(11,35)$ & $48,54(13,45)$ & $64,04(15,79)$ \\
SRQ-20 & $2,86(2,19)$ & $11,05(2,58)$ & $4,46(2,12)$ & $12,38(3,29)$ \\
ASRS-19(HI) & $11,49(4,89)$ & $13,84(4,54)$ & $20,35(6,14)$ & $21,64(6,05)$ \\
ASRS-18 (D) & $12,72(4,59)$ & $15,72(4,75)$ & $21,73(6,38)$ & $24,38(5,90)$ \\
\hline
\end{tabular}

p 0,001; CFQ: Questionário de Falhas Cognitivas; SRQ-20: Self-Reporting Questionnaire 20; ASRS-18: Adult Self-Reported Scale 18; HI: hiperatividade-impulsividade; D: desatenção.
Quando os quatro grupos são comparados em termos de falhas cognitivas, obtemos um modelo significativo $(F=71,36, p<0,001)$. As análises post hoc sugerem que o grupo controle apresentou menos falhas cognitivas que o grupo internalizante ( $p<0,001, d=0,86)$, externalizante $(p<0,001, d=1,00)$ e misto $(p<0,001, d=2,01)$. Os grupos internalizante e externalizante não apresentaram diferenças estatisticamente significativas entre si ( $p=0,800, d=0,21)$, mas ambos apresentaram menos falhas quando comparados ao grupo misto $(p<0,001, d=1,31$ e $p<0,001, d=1,05$, respectivamente). A Figura 1 sintetiza essas comparações.

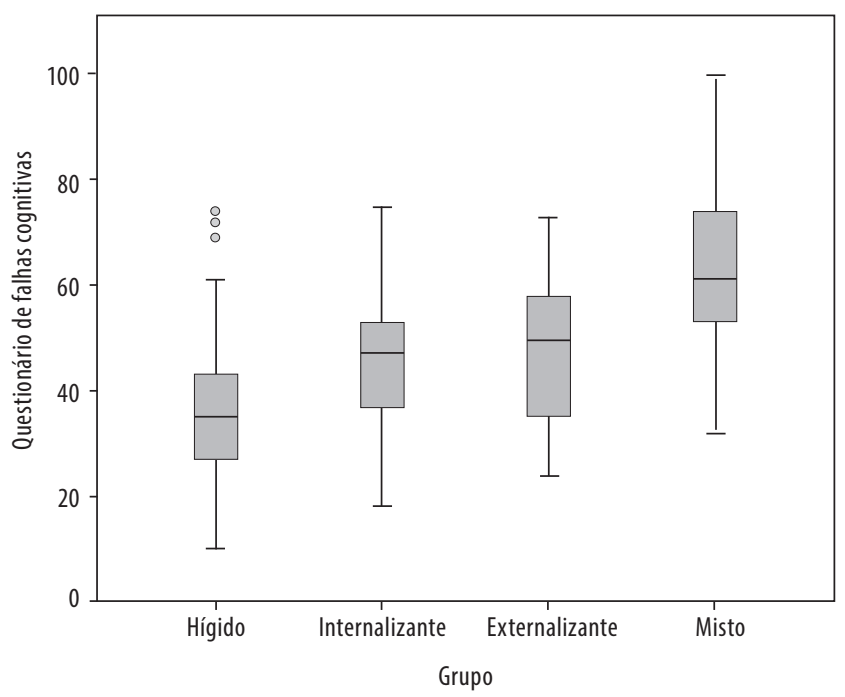

Figura 1. Falhas cognitivas em participantes hígidos e nos grupos clínicos avaliados.

\section{DISCUSSÃO}

Nossos resultados sugerem que tanto sintomas internalizantes quanto externalizantes se correlacionam positivamente com a ocorrência de falhas cognitivas no dia a dia. Quando os participantes foram estratificados em grupos, com base na prevalência de sintomas externalizantes, internalizantes ou ambos, observamos que todos os grupos apresentam mais falhas cognitivas que o grupo controle. Além disso, os grupos internalizantes e externalizantes não diferiram entre si, porém a combinação dos dois sintomas no grupo misto gera um padrão mais intenso de falhas cognitivas, superior a todos os demais grupos estudados. Nossos resultados sugerem que tanto sintomas internalizantes quanto sintomas externalizantes se associam às falhas cognitivas. Mais ainda, ambos os transtornos parecem apresentar um efeito sinérgico, visto que, em conjunto, comprometem ainda mais o funcionamento cognitivo dos participantes no dia a dia.

A associação de sintomas externalizantes com as falhas cognitivas é a mais comumente estudada na literatura, ten- 
do em vista que, por definição, queixas de desatenção e impulsividade por exemplo são tanto sintomas externalizantes como manifestações cognitivas ${ }^{6,22}$. Já a associação dos sintomas internalizantes com queixas cognitivas é menos intuitiva, uma vez que os sintomas desse tipo em geral se relacionam ao humor e à regulação emocional. Contudo, vários estudos sugerem que sintomas internalizantes se associam ao funcionamento cognitivo, quer em termos de autorrelato ${ }^{4}$ ou de avaliação cognitiva por meio de testes ${ }^{7}$. Embora esses dois aspectos anteriores estejam bem documentados na literatura, a comparação de um grupo misto, tanto com sintomas internalizantes quanto externalizantes, é raramente estudada. Nesse sentido, nosso estudo traz uma contribuição interessante ao campo, sugerindo que ambos os sintomas contribuem para o ocorrência de falhas cognitivas no dia a dia. Isso indica que as falhas cognitivas no dia a dia podem ser um desfecho comum a quadros clínicos de etiologia distinta, e é corroborado pela similaridade das queixas cognitivas entre os grupos internalizante e externalizante o efeito aditivo desses dois sintomas na ocorrência das falhas.

Esse achado pode ter implicações importantes em relação à terapêutica das falhas cognitivas. Sintomas internalizantes e externalizantes ocorrem em diversos transtornos mentais, podendo ainda coocorrer em um mesmo paciente. Pacientes com queixas cognitivas associadas a sintomas internalizantes ou externalizantes poderiam apresentar meIhora cognitiva após o tratamento dos transtornos em si23,24. Contudo, o tipo de tratamento adotado para transtornos internalizantes usualmente difere do dos transtornos externalizantes, em termos tanto farmacológicos quanto psicoterápicos ou mesmo neuromodulação, sugerindo que múltiplos tratamentos podem levar a um mesmo desfecho em função da provável causa das falhas cognitivas. Nos participantes com sintomas internalizantes e externalizantes concomitantes, terapêuticas distintas podem ser combinadas e possivelmente apresentar sinergia na redução das falhas cognitivas. Esse tópico pode ser melhor investigado em futuros estudos.

Carrigan e Barkus ${ }^{3}$ argumentam, em um artigo de revisão, que a ocorrência de falhas cognitivas é uma variável do tipo "estado". Isso sugere que, embora o participante possua um perfil cognitivo relativamente estável (traço), que é mensurado por exemplo por meio de testes psicológicos ou neuropsicológicos, nem sempre é possível que ele aplique suas habilidades integralmente no dia a dia. Sintomas internalizantes e externalizantes podem ser variáveis moderadoras dessa relação, dificultando a aplicação dos recursos cognitivos em situações de vida real. Isso explicaria a associação relativamente baixa entre os testes cognitivos e as falhas cognitivas no dia a dia e sua associação significativamente maior com a saúde mental ${ }^{25,26}$.

O presente estudo apresenta limitações que devem ser consideradas em sua interpretação. Nossos participantes compuseram uma amostra de conveniência, não representando a população adulta brasileira em termos epidemiológicos. Como os dados foram coletados em um ambiente virtual, a temática e o acesso à plataforma de pesquisa constituem um viés de seleção dos participantes. Avaliamos ainda apenas o autorrelato dos participantes por meio de questionários, não sendo realizada, nessa amostra, avaliação presencial ou propriamente uma entrevista diagnóstica para transtornos mentais. Contudo, algumas de suas características mais positivas envolvem um tamanho amostral suficiente para a detecção dos efeitos investigados, o uso de questionários e escalas adaptados e validados no contexto brasileiro e o uso do CFQ, considerado ainda hoje como a medida mais robusta de falhas cognitivas no dia a dia.

\section{CONCLUSÃO}

O estudo sugere que as falhas cognitivas se associam tanto a sintomas internalizantes quanto externalizantes em participantes adultos. A concorrência desses sintomas apresenta um efeito sinérgico nas falhas cognitivas, aumentando ainda mais sua frequência.

\section{CONFLITOS DE INTERESSE}

Os autores declaram não possuir conflitos de interesse em relação a este estudo.

\section{CONTRIBUIÇÕES INDIVIDUAIS}

Aline Alves Ferreira e Wanessa Gabrielli Augusto Oliveira realizaram a coleta dos dados. Todos os autores contribuíram para a análise dos dados, redação e revisão do artigo, e aprovaram sua versão final.

\section{AGRADECIMENTOS}

Aos representantes do Comitê de Ética em Pesquisa (CEP) da Faculdade de Ciências Médicas de Minas Gerais (FCMMG), pelas orientações e auxílio durante as fases preliminares dessa pesquisa, e à Fundação de Amparo à Pesquisa do Estado de Minas Gerais (Fapemig), pelo apoio financeiro à iniciação científica das duas primeiras autoras. Agradecemos, ainda, à professora Kathy R. Parkes, por nos autorizar a adaptar o QFC ao Brasil e a utilizá-lo em nossas pesquisas.

\section{REFERÊNCIAS}

1. Deary IJ, Penke L, Johnson W. The neuroscience of human intelligence differences. Nat Rev Neurosci. 2010;11(3):201-11. 
2. Broadbent DE, Cooper PF, FitzGerald P, Parkes KR. The Cognitive Failures Questionnaire (CFO) and its correlates. Br J Clin Psychol. 1982;21 (Pt 1):1-16.

3. Carrigan N, Barkus E. A systematic review of cognitive failures in daily life: Healthy populations. Neurosci Biobehav Rev. 2016;63:29-42.

4. Payne TW, Schnapp MA. The relationship between negative affect and reported cognitive failures. Depress Res Treat. 2014;2014:396195.

5. Abbasi M, Bagyan MJ, Dehghan H. Cognitive failure and alexithymia and predicting high - Risk behaviors of students with learning disabilities. Int J High Risk Behav Addict. 2014;3(2): e16948.

6. Carragher N, Krueger RF, Eaton NR, Slade T. Disorders without borders: current and future directions in the meta-structure of mental disorders. Soc Psychiatry Psychiatr Epidemiol. 2015;50(3):339-50

7. de Paula JJ, Diniz BS, Bicalho MA, Albuquerque MR, Nicolato R, de Moraes EM, et al. Specific cognitive functions and depressive symptoms as predictors of activities of daily living in older adults with heterogeneous cognitive backgrounds. Front Aging Neurosci. 2015;7:139.

8. Costa DS, Paula JJ, Alvim-Soares Júnior AM, Diniz BS, Romano-Silva MA, Malloy-Diniz LF, et al. ADHD inattentive symptoms mediate the relationship between intelligence and academic performance in children aged 6-14. Rev Bras Psiquiatr. 2014;36(4):313-21.

9. Brown PJ, Liu X, Sneed JR, Pimontel MA, Devanand DP, Roose SP. Speed of processing and depression affect function in older adults with mild cognitive impairment. Am J Geriatr Psychiatry. 2013;21(7):675-84.

10. Das D, Cherbuin N, Butterworth P, Anstey KJ, Easteal S. A population-based study of attention deficit/hyperactivity disorder symptoms and associated impairment in middle-aged adults. PLoS One. 2012;7(2):e31500

11. Erskine HE, Norman RE, Ferrari AJ, Chan GC, Copeland WE, Whiteford HA, et al. Long-term outcomes of attention-deficit/hyperactivity disorder and conduct disorder: a systematic review and meta-analysis. J Am Acad Child Adolesc Psychiatry. 2016;55(10):841-50.

12. Oquendo M, Brent DA, Birmaher B, Greenhill L, Kolko D, Stanley B, et al. Posttraumatic stress disorder comorbid with major depression: factors mediating the association with suicidal behavior. Am J Psychiatry. 2005;162(3):560-6.

13. de Paula JJ. Propriedades psicométricas do Índice de Religiosidade de Duke aplicado em plataforma virtual. Cad Saúde Coletiva. 2015;23(3):276-9.
14. Faul F, Erdfelder E, Lang AG, Buchner A. G*Power 3: a flexible statistical power analysis program for the social, behavioral, and biomedical sciences. Behav Res Methods. 2007;39(2):175-91.

15. Bridger RS, Johnsen SÅ, Brasher K. Psychometric properties of the Cognitive Failures Questionnaire. Ergonomics. 2013;56(10):1515-24.

16. de Paula JJ, Costa DS, Miranda DM, Romano-Silva MA. Brazilian version of the Cognitive Failures Questionnaire (CFO): cross-cultural adaptation and evidence of validity and reliability. Rev Bras Psiquiatr. 2017. [no prelo]

17. Santos KOB, Araújo TM, Oliveira NF. Estrutura fatorial e consistência interna do Self-Reporting Questionnaire (SRQ-20) em população urbana. Cad Saúde Pública. 2009:25(1):214-22.

18. Gonçalves DM, Stein AT, Kapczinski FP. Avaliação de desempenho do Self-Reporting Questionnaire como instrumento de rastreamento psiquiátrico: um estudo comparativo com 0 Structured Clinical Interview for DSM-IV-TR. Cad Saúde Pública. 2008;24(2):380-90.

19. Mattos P, Segenreich D, Saboya E, Louzã M, Dias G, Romano M. Adaptação transcultural para o português da escala Adult Self-Report Scale para avaliação do transtorno de déficit de atenção/hiperatividade (TDAH) em adultos. Rev Psiquiatr Clín. 2006;33(4):188-94.

20. Leite WB. Avaliação das propriedades psicométricas da Escala de Autorrelato de Sintomas do Transtorno do Déficit de Atenção e Hiperatividade - ASRS-18 [dissertação]. Belo Horizonte: Universidade Federal de Minas Gerais; 2010.

21. JASP Team. JASP (version 0.8.0.0) [computer software]. 2016.

22. Rast P, Zimprich D, Van Boxtel M, Jolles J. Factor structure and measurement invariance of the cognitive failures questionnaire across the adult life span. Assessment. 2009;16(2):145-58.

23. Mandelli L, Serretti A, Colombo C, Florita M, Santoro A, Rossini D, et al. Improvement of cognitive functioning in mood disorder patients with depressive symptomatic recovery during treatment: an exploratory analysis. Psychiatry Clin Neurosci. 2006;60(5):598-604.

24. Pearson DA, Lane DM, Santos CW, Casat CD, Jerger SW, Loveland KA, et al. Effects of methylphenidate treatment in children with mental retardation and $\mathrm{ADHD}$ : individual variation in medication response. J Am Acad Child Adolesc Psychiatry. 2004;43(6):686-98.

25. Paiva GCC, Fialho MB, Costa DS, de Paula JJ. Ecological validity of the five digit test and the oral trails test. Arq Neuropsiquiatr. 2016;74(1):29-34.

26. Zlatar ZZ, Moore RC, Palmer BW, Thompson WK, Jeste DV. Cognitive complaints correlate with depression rather than concurrent objective cognitive impairment in the successful aging evaluation baseline sample. J Geriatr Psychiatry Neurol. 2014;27(3):181-7. 